Revista Matemática Universitária, vol. 2, 2021

ISSN: 2675-5254 — DOI: 10.21711/26755254/rmu20216

\title{
A ESPIRAL LOGARÍTMICA E O LOGO DA SBM
}

\author{
MICHEL SPIRA
}

\begin{abstract}
Resumo. A espiral logarítmica é uma das mais fascinantes curvas da Matemática, devido a suas maravilhosas propriedades e sua ubiquidade em fenômenos naturais e científicos. Ela foi objeto de estudo de, entre outros, Descartes, Torricelli e Jacob Bernoulli. Aqui apresentamos a família de espirais logarítmicas e algumas de suas propriedades, mostramos como gerar espirais logarítmicas a partir de retângulos e triângulos isósceles, e determinamos quando as espirais assim geradas são tangentes aos lados da figura geradora. Ao final, discutimos o logo da SBM.
\end{abstract}

\section{A ESPIRAL LOGARÍtMicA}

Uma curva dada em um sistema de coordenadas polares de origem $O$ por uma equação da forma

$$
r(\theta)=a p^{\theta}
$$

onde $a, p \in \mathbb{R}^{+}$é dita uma espiral logarítmica de centro $O$. Essa equação determina uma família $\mathcal{L}$ de curvas a dois parâmetros; $a=r(0)$ é apenas um fator de escalonamento e não tem influência no comportamento assintótico de uma espiral logarítmica, que é determinado por $p$. Quando $p<1$ temos $\lim _{\theta \rightarrow \infty} r(\theta)=0$ e quando $p>1$ temos $p<1$ temos $\lim _{\theta \rightarrow-\infty} r(\theta)=0$; em ambos os casos, podemos considerar $O$ como uma representação do infinito.

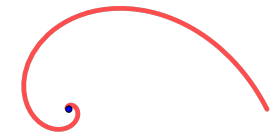

$$
p=0,6
$$

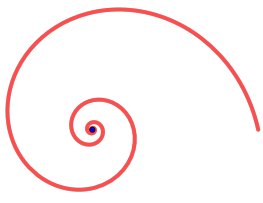

$p=0,8$

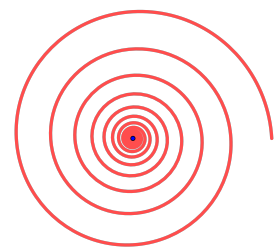

$p=0,95$

Data de aceitação: Junho de 2021.

Palavras chave. Espiral logarítmica, logo da SBM. 


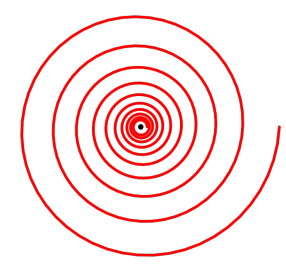

$p=1,05$

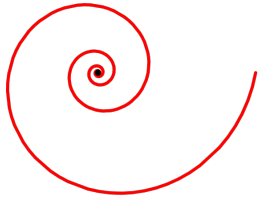

$p=1,2$

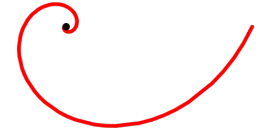

$p=1,6$

Definimos acima uma família de curvas, mas mesmo assim vamos ocasionalmente referir-nos "à" espiral logarítmica. Esse abuso de linguagem já é consagrado e seu uso indica que discurso está sendo feito de modo genérico.

O centro $O$ e dois pontos distintos $(b, \beta)$ e $(c, \gamma)$ em coordenadas polares com $b, c \in \mathbb{R}^{+}$determinam uma única espiral logarítmica. De fato, definindo

$$
p=\left(\frac{b}{c}\right)^{\frac{1}{\beta-\gamma}}
$$

e

$$
a:=\frac{b}{p^{\beta}}=\frac{c}{p^{\gamma}} .
$$

temos que a espiral logarítmica dada por $r=a p^{\theta}$ passa pelos pontos dados. Reciprocamente, quando conhecidos o centro e dois pontos (também em coordenadas polares) de uma mesma espiral logarítmica, os parâmetros $a$ e $p$ podem ser recuperados como acima.

Para finalizar essa introdução, observamos que as funções $r$ como em (1) levam progressões aritméticas em progressões geométricas e são as únicas funções com essa propriedade; equivalentemente, suas inversas são as únicas funções que levam progressões geométricas em progressões aritméticas ([1], capítulo 8). Dessa maneira, as ideias de raios igualmente espaçados angularmente e raios em progressão geométrica são equivalentes em uma espiral logarítmica.

1.1. A propriedade equiangular. Uma linda propriedade de $\mathcal{L}$ é que o ângulo entre o raio vetor e a tangente em qualquer ponto de uma espiral logarítmica dada é constante; vamos denotar esse ângulo por $\delta$ a partir de agora.

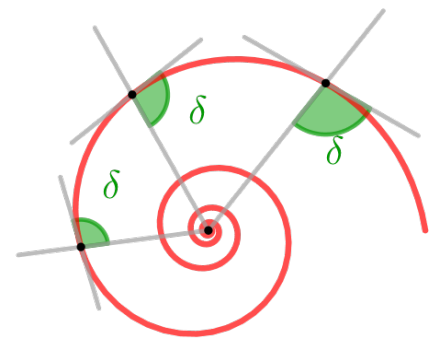

Do ponto de vista qualitativo, isso decorre imediatamente do fato apontado anteriormente de que $r$ como em (1) leva progressões aritméticas em progressões geométricas; para nossos propósitos, no entanto, é importante caracterizar $\delta$ quantitativamente, 
o que vamos fazer mostrando que $\operatorname{ctg} \delta \equiv \ln p$. Para isso, consideremos a figura abaixo, onde supomos por um momento que $r$ é uma função diferenciável qualquer ${ }^{1}$.

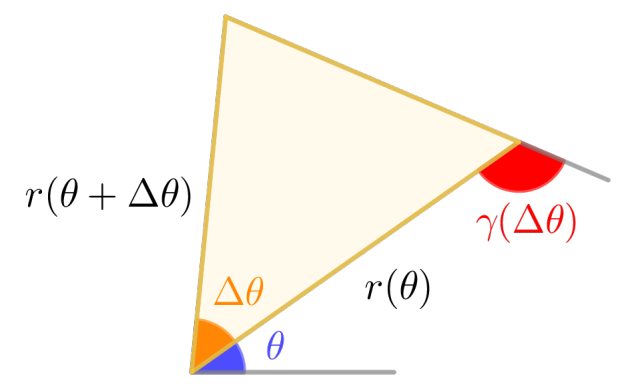

A lei dos senos nos dá a primeira igualdade abaixo

$$
\frac{r(\theta+\Delta \theta)}{\operatorname{sen} \gamma(\Delta \theta)}=\frac{r(\theta)}{\operatorname{sen}[\gamma(\Delta \theta)-\Delta \theta]}=\frac{r(\theta+\Delta \theta)-r(\theta)}{\operatorname{sen} \gamma(\Delta \theta)-\operatorname{sen}[\gamma(\Delta \theta)-\Delta \theta]}
$$

e a segunda segue de propriedades elementares de proporções. Reescrevendo essa última igualdade como

$$
\frac{r(\theta+\Delta \theta)-r(\theta)}{\Delta \theta} \cdot \frac{1}{r(\theta)}=\frac{\operatorname{sen} \gamma(\Delta \theta)-\operatorname{sen}[\gamma(\Delta \theta)-\Delta \theta]}{\Delta \theta} \cdot \frac{1}{\operatorname{sen}[\gamma(\Delta \theta)-\Delta \theta]}
$$

fazendo $\Delta \theta \rightarrow 0$ e observando que $\lim _{\Delta \theta \rightarrow 0} \gamma(\Delta \theta)=\delta(\theta)$ obtemos

$$
\frac{r^{\prime}(\theta)}{r(\theta)}=\operatorname{ctg} \delta(\theta)
$$

Quando $r(\theta)=a p^{\theta}$ temos $\operatorname{ctg} \delta(\theta)=\ln p$ para todo $\theta$, como anunciado. A equação (1) costuma ser apresentada na forma $r(\theta)=a e^{b \theta}$ para deixar em evidência $o$ parâmetro $b=\ln p$.

Reciprocamente, (2) mostra que a propriedade equiangular é característica de $\mathcal{L}$; por esse motivo, uma espiral logarítmica também atende pelo nome de espiral equiangular.

1.2. Autosimilaridade. Outra bela propriedade de $\mathcal{L}$ é a autosimilaridade por rotação, que diz que uma rotação com centro $O$ e ângulo $\alpha$ e uma dilatação de centro $O$ e razão $p^{\alpha}$ têm o mesmo efeito sobre uma espiral logarítmica. Para ver isso, basta escrever $r(\theta+\alpha)=a p^{\theta+\alpha}=p^{\alpha} r(\theta)$ para todo $\theta$.

\footnotetext{
${ }^{1} \mathrm{~A}$ redação original do argumento que segue foi corrigida e simplificada com sugestões dos relatores.
} 


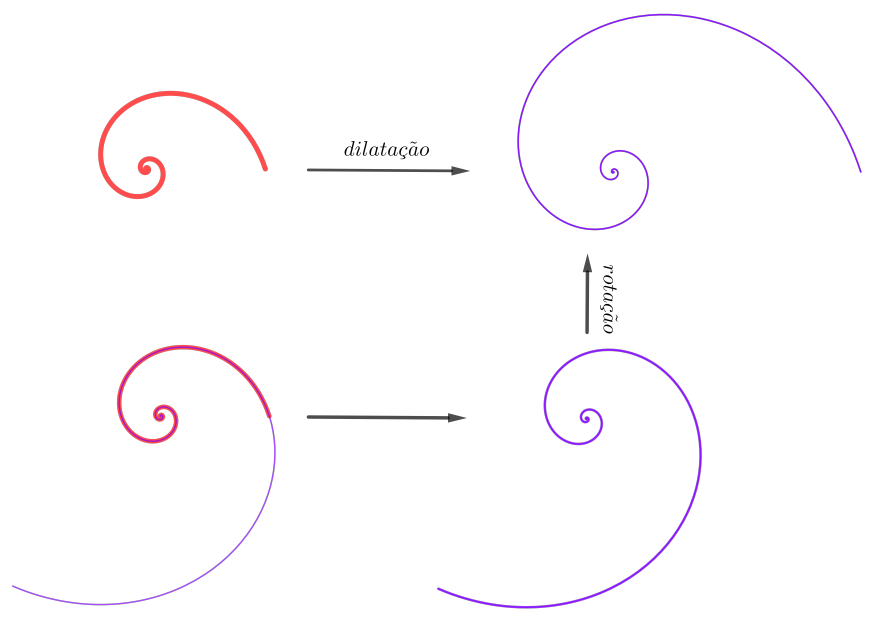

A autosimilaridade por rotação é uma propriedade característica de $\mathcal{L}$. De fato ${ }^{2}$, seja $r$ uma curva contínua dada em coordenadas polares tal que para todo $\alpha$ existe $\lambda(\alpha)$ tal que

$$
\lambda(\alpha) r(\theta)=r(\theta+\alpha)
$$

para todo $\theta$; sem perda de generalidade, podemos supor $r(0)=1$. Colocando $\theta=0$ em (3) obtemos $\lambda(\alpha)=r(\alpha)$ para todo $\alpha$; em particular, $\lambda$ é contínua. Segue também de (3) que $\lambda(\alpha+\beta)=r(\alpha+\beta)=\lambda(\alpha) \lambda(\beta)$ para todos $\alpha, \beta$ e logo $\lambda(\alpha)=e^{c \alpha}$ para algum $c$ ([1], capítulo 8). Colocando $p=e^{c} \operatorname{temos} r(\theta)=\lambda(\theta)=e^{c \theta}=p^{\theta}$ e recuperamos uma espiral logarítmica.

1.3. Um mínimo de História. Vamos agora apresentar alguns aspectos históricos da espiral logarítmica.

René Descartes (1596-1650) descobriu a espiral logarítmica a partir da propriedade equiangular; devemos a ele a terminologia espiral equiangular. A primeira menção a essa curva aparece em uma de suas cartas, enviada para Marin Mersenne (1588-1648) em 1638 [2].

Evangelista Torricelli (1608-1647) determinou em 1645 o comprimento da espiral logarítmica de $\theta=0$ a $\theta=\infty$ usando o método de exaustão ([2],[3] capítulo 9). Essa foi a segunda retificação de uma curva na história da Matemática, a primeira sendo a da circunferência. O resultado de Torricelli aparece na figura abaixo.

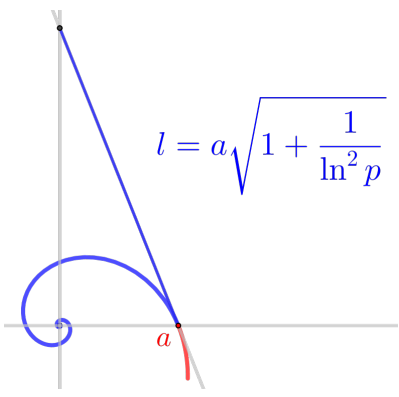

${ }^{2}$ Argumento sugerido por Marco Moriconi. 
Jacob Bernoulli (1654-1705) estudou profundamente a espiral logarítmica e descobriu muitas de suas maravilhosas propriedades, que o levaram a chamá-la de spira mirabilis. Descrever as contribuições de Bernoulli foge ao escopo desse artigo; observamos apenas que a terminologia espiral logarítmica foi introduzida por ele em 1691 [2].

Mencionamos também que Isaac Newton (1642-1726) mostrou nos Principia que se um corpo percorre uma espiral logarítmica sob a ação de uma força central então essa força varia com o inverso do cubo da distância ao centro $([4],[5])$.

Encerramos aqui nossa breve apresentação da espiral logarítmica. Recomendamos ([2],[3] capítulo 9) para exposições históricas mais completas, ([6],[7]) para detalhes sobre as propriedades que tanto encantaram Bernoulli, ([8],[9],[10]) para suas manifestações em outras áreas de conhecimento, ([11],[12],[13]) para informações adicionais e lindas figuras, e [14] para os interessados em seus aspectos místicos.

\section{A ESPiral ASSOCiAdA A UM REtÂNGUlo}

A figura abaixo, à esquerda, mostra um retângulo $A B C D$ de dimensões $\ell \times 1 \mathrm{com}$ $\ell>1$; o ponto $O$ é a interseção da diagonal $B D$ com a perpendicular por $C$.
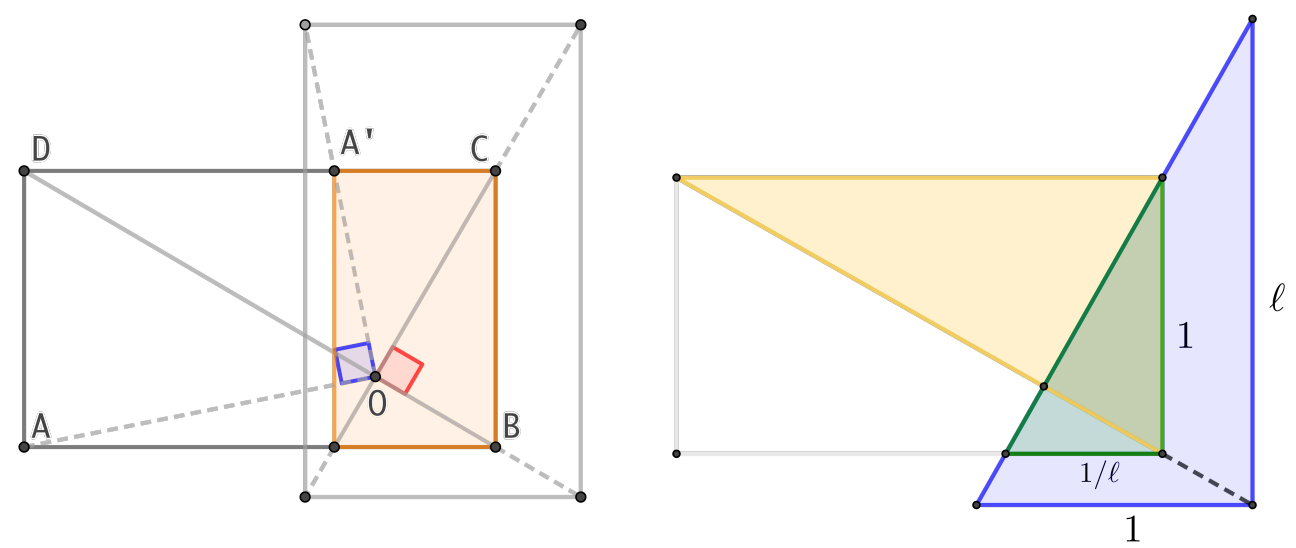

O retângulo sombreado é obtido do retângulo original por uma rotação de $\frac{\pi}{2}$ em torno de $O$ no sentido horário seguida de uma contração de $\frac{1}{\ell} \operatorname{com}$ centro $O$; o ponto $A^{\prime}$ é a imagem de $A$ por esse processo e $O A$ é perpendicular a $O A^{\prime}$. Para justificar essas afirmativas ${ }^{3}$ basta observar a figura à direita, onde notamos que todos os triângulos que aparecem são semelhantes. O triângulo azul é a imagem do triângulo amarelo pela rotação e o triângulo verde, a imagem desse último pela contração. Como a imagem do retângulo original por essas transformações é um retângulo e já temos as imagens de três de seus vértices, a correção da figura à esquerda fica estabelecida.

Como o retângulo sombreado é semelhante ao retângulo original, podemos iterar essa construção e obter a figura a seguir, à esquerda, onde exibimos cinco iterações, as sucessivas imagens de $A$ e os raios por elas determinados. Esses raios formam uma progressão geométrica de razão $\frac{1}{\ell}$ e estão angularmente espaçados de $\frac{\pi}{2}$, de

\footnotetext{
${ }^{3}$ Agradecemos a um dos relatores por insistir na inclusão de uma justificativa.
} 
modo que seus extremos estão em uma espiral de centro $O$, como à direita. Dizemos que essa é a espiral associada ao retângulo; para ela temos $p=\ell^{\frac{2}{\pi}}$.
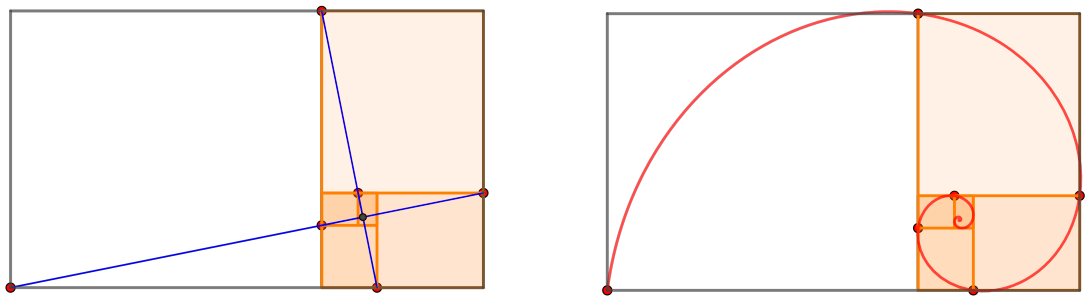

A figura a seguir
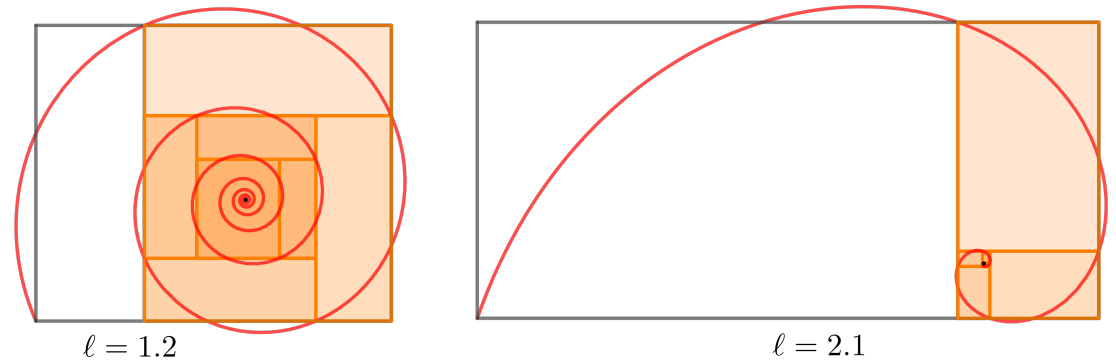

e o teorema do valor intermediário mostram que existe um único retângulo $\ell_{0} \times 1$ tal que a espiral associada é tangente ao lado $C D$ (e logo também tangente a $B C$ e $A B)$. Para determinar $\ell_{0}$, consideramos o retângulo $\ell \times 1$ da figura abaixo

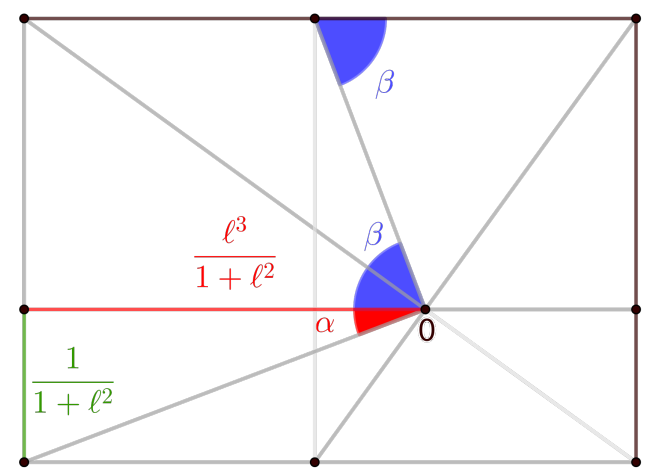

onde vemos que $\operatorname{ctg} \beta=\operatorname{tg} \alpha=\frac{1}{\ell^{3}}$. A condição de tangência é $\beta=\delta ; \operatorname{como} \operatorname{ctg} \delta=$ $\ln p$ temos, nesse caso,

$$
\frac{1}{\ell^{3}}=\frac{2}{\pi} \ln \ell
$$

e segue que $\ell_{0}$ é o (único) zero de $f(x)=x^{3} \ln x-\frac{\pi}{2}$. O comando Root do Geogebra fornece $\ell_{0}=1,5388620467 \ldots 4$; aqui temos $\delta_{0}=74^{\circ} 39^{\prime} 18^{\prime \prime} \ldots$. Segue para contemplação a figura correspondente.

${ }^{4} \mathrm{O}$ Mathematica fornece o mesmo valor, como informa um dos relatores. 


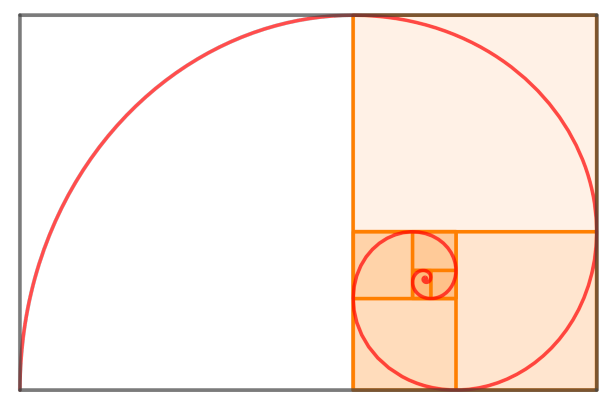

\section{A ESPIRAL ASSOCIADA A UM TRIÂNGULO ISÓSCELES}

Espirais logarítmicas também aparecem associadas a triângulos isósceles, em particular ao assim dito triângulo áureo ([15],[16]). Vamos aqui analisar brevemente essas espirais, de modo análogo ao que fizemos na seção anterior.

Consideremos um triângulo isósceles $A B C$ de base $B C=1$ e lados $A B=A C=\ell$, com $\widehat{A B C}=\widehat{A C B}=\alpha$; nele determinamos o ponto $O$ como a interseção de arcos capazes de $\pi-\alpha$ dos segmentos $A B$ e $B C$.

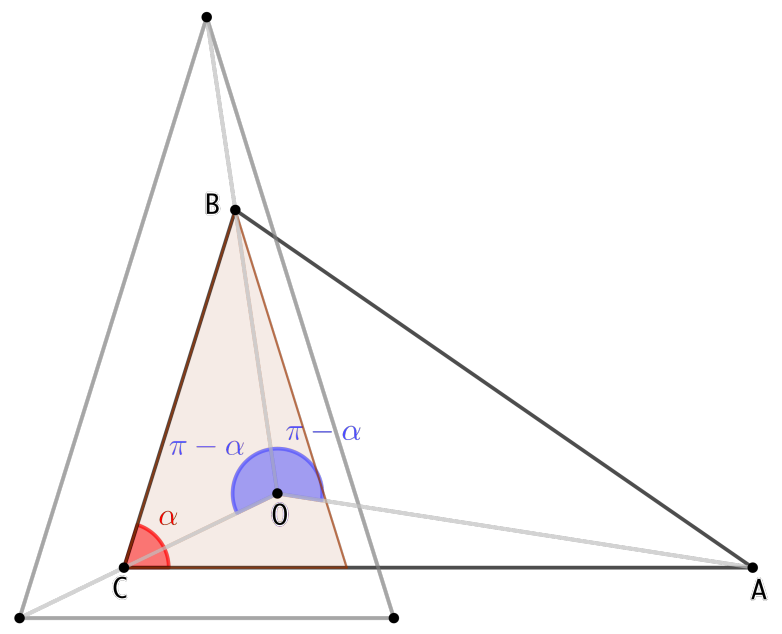

O triângulo sombreado é obtido a partir do triângulo original por uma rotação de $\pi-\alpha$ em torno de $O$ no sentido anti-horário seguida de uma contração de $\frac{1}{\ell}$ com centro $O$; a iteração desse processo leva a uma espiral logarítmica de centro $O$ que passa pelas sucessivas imagens de $A$.
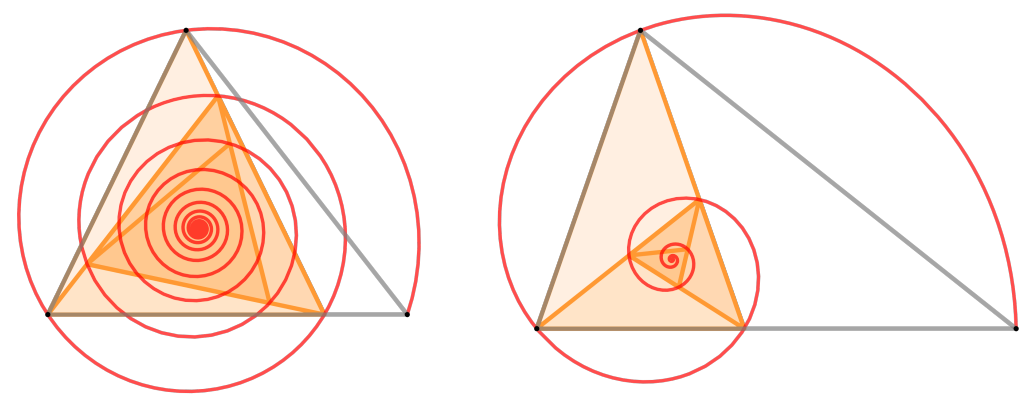
Para essa espiral temos $p=\left(\frac{1}{\ell}\right)^{\frac{1}{\pi-\alpha}}$. Como na seção anterior, existe um único triângulo isósceles cuja espiral associada é tangente a seus lados. Para determiná-lo, consideremos a seguinte figura.

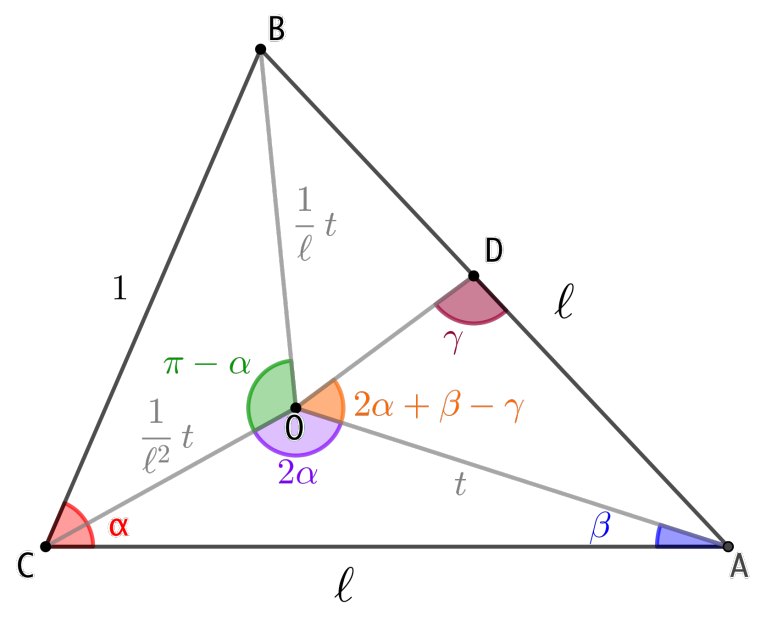

Aqui $D$ é o primeiro ponto (quando existe) onde a espiral associada intercepta o lado $A B$; a diferença angular entre $A$ e $D$ é $2 \pi+2 \alpha+\beta-\gamma$. Temos

$$
\begin{gathered}
\alpha=\arccos \frac{1}{2 \ell} \\
t=\ell^{2} \sqrt{\frac{1}{\ell^{2}+2}}(\text { lei dos cossenos no triângulo } A C O) \\
\beta=\operatorname{arcsen} \frac{t \operatorname{sen} 2 \alpha}{\ell^{3}}(\text { lei dos senos no triângulo } A C O) \\
O D=t p^{2 \pi+2 \alpha+\beta-\gamma}
\end{gathered}
$$

e lembramos que

$$
\delta=\operatorname{arcctg} \ln p=\arccos \frac{\ln p}{\sqrt{1+\ln ^{2} p}} .
$$

A espiral é tangente em $D$ quando $\gamma=\delta$; nesse caso, a lei dos senos no triângulo $A D O$ nos dá

$$
\frac{t}{\operatorname{sen} \delta}=\frac{t p^{2 \pi+2 \alpha+\beta-\delta}}{\operatorname{sen}(2 \alpha+\beta)}
$$

ou seja

$$
\operatorname{sen}(2 \alpha+\beta)=p^{2 \pi+2 \alpha+\beta-\delta} \operatorname{sen} \delta
$$

e segue que a tangência ocorre quando $\ell$ é o (único) zero da função

$$
g(x)=\operatorname{sen}[2 \alpha(x)+\beta(x)]-p(x)^{2 \pi+2 \alpha(x)+\beta(x)-\delta(x)} \operatorname{sen} \delta(x) .
$$

O comando Root fornece o zero $\ell_{1}=1,2162407940 \ldots{ }^{5}$, que corresponde a um triângulo isósceles com ângulo da base $65^{\circ} 43^{\prime} 33^{\prime \prime} \ldots$ e a $\delta_{1}=96^{\circ} 10^{\prime} 21^{\prime \prime} \ldots$. Segue, também para contemplação, a figura pertinente.

${ }^{5}$ Já o Mathematica fornece $\ell_{1}=1,2162407942 \ldots$, como apontado pelo mesmo relator. 


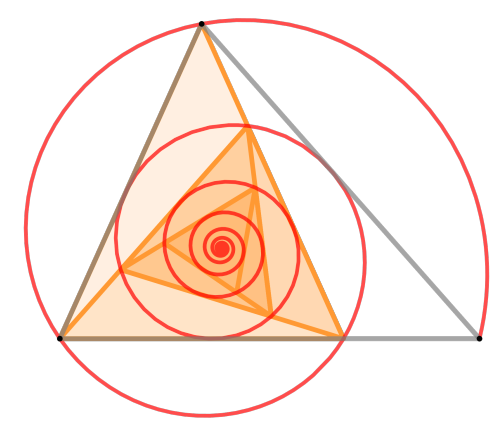

\section{O LOGO DA SBM}

Nessa seção vamos considerar alguns aspectos do logo da SBM, começando por sua história.

Um concurso para a escolha do logo foi divulgado no Noticiário da SBM em outubro de 1977. Em reunião do Conselho Diretor de maio de 1978 não houve consenso sobre nenhuma das propostas, o que se repetiu em em reuniões de março e outubro de 1980, bem como de junho de 1981; a partir daí não se encontram mais menções a esse assunto no Noticiários $[17]^{6}$. Em comunicação pessoal, o professor César Camacho informa que o logo foi escolhido em um concurso realizado pela diretoria da SBM durante seu mandato como presidente no período 1987-89; a proposta vencedora foi de Rodolfo Capeto, na época o designer gráfico do IMPA.

A descrição do logo aparece no Manual da marca e identidade visual da SBM [18]

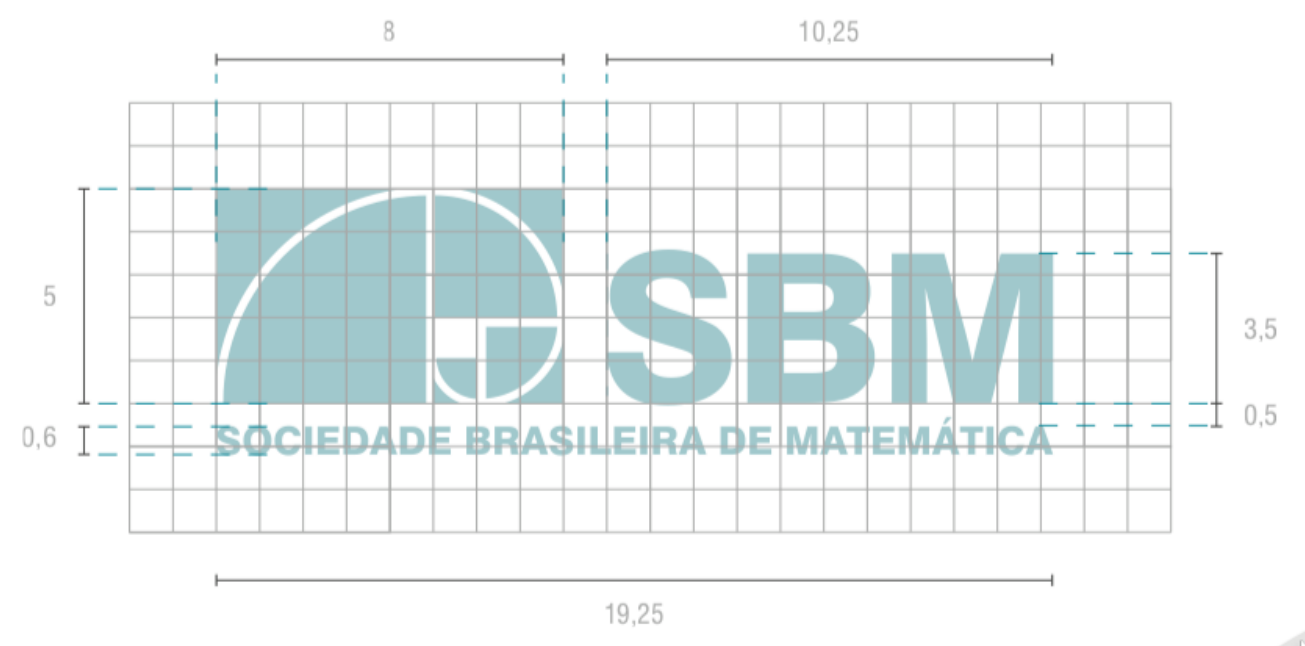

e vemos que ele é a conhecida espiral de Fibonacci ([19],[20]) em um retângulo $8 \times 5$. A partir do quadrado branco, os lados dos quadrados são 1, 1, 2, 3 e 5 que, com o lado 8 do retângulo, são os primeiros termos da sequência de Fibonacci. Essa espiral é formada por quartos de círculo ${ }^{7}$, de modo a fazê-la tangente aos lados do retângulo.

\footnotetext{
${ }^{6}$ Referência indicada por Paulo Cézar Carvalho.

${ }^{7} \mathrm{O}$ Manual não especifica a espessura dos arcos de círculo e dos segmentos que delimitam os quadrados; em uma grade apropriada, observa-se que ela é aproximadamente $\frac{1}{5}$.
} 

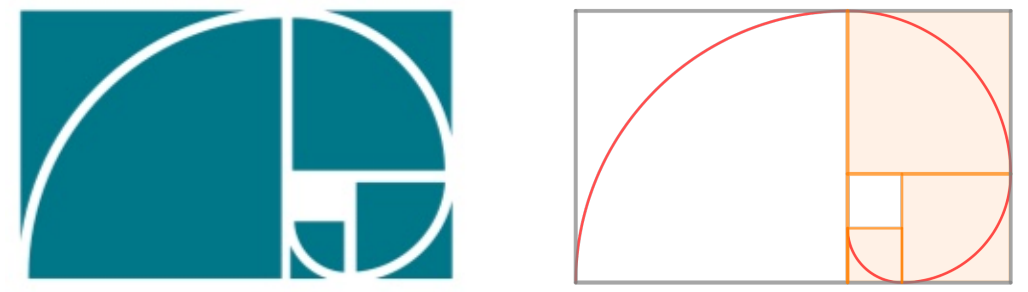

Como $\frac{8}{5 \Phi}=0,9888 \ldots$, o retângulo $\frac{8}{5} \times 1$ é uma boa aproximação do retângulo $\Phi \times 1$, onde $\Phi=\frac{1+\sqrt{5}}{2}$ é o número de ouro. Retângulos semelhantes a esse último, conhecidos como retângulos áureos, são os únicos retângulos que deixam quadrados em seu caminho no processo de rotação e contração descrito na seção 2.

Podemos assim visualizar no logo as ideias de quadrado, círculo, tangência e números de Fibonacci e, de modo aproximado, do número de ouro e do retângulo áureo. Ressaltamos também a simplicidade de seus elementos, que resulta em grande facilidade para sua descrição e composição gráfica.

Por outro lado, a gênese da espiral de Fibonacci através de arcos de círculo e números de Fibonacci faz com que ela não seja sequer de classe $C^{2}$ e exclui qualquer sugestão das ideias de autosimilaridade, equiangularidade e representação do infinito. Notamos que, com exceção da referência a números de Fibonacci, ela não tem relação com a História da Matemática, e observamos ainda que, às vezes apresentada como uma espiral logarítmica, ela ilustra o uso incorreto e autoritário de linguagem matemática em pseudociência, misticismo, culto do número de ouro, arte e outras manifestações culturais ([14],[21],[22],[23],[24]).

A construção de uma espiral por meio de quartos de círculo pode ser realizada no retângulo áureo ([16],[25]) e é originalmente devida a Albrecht Dürer (1471-1528) [20]. Aqui aplicam-se os mesmos comentários feitos no parágrafo anterior em relação à espiral de Fibonacci, com exceção da possibilidade de representação do infinito.

Notamos, finalmente, que essa não é a primeira vez que o logo de uma sociedade matemática é criticado ${ }^{8}$. Em [26] (ver também [27]) aponta-se que o logo da MAA anterior a 1972 apresentava uma projeção em perspectiva incorreta do icosaedro, que foi devidamente corrigida.

\section{Duas propostas}

O logo da SBM acompanhou boa parte da história da Matemática brasileira, tendo assim inegável valor simbólico e afetivo. Por outro lado, de acordo com o exposto na seção anterior, consideramos que ele não possui características adequadas para representar nossa Sociedade. Desse modo, apresentamos duas propostas para eventual consideração por parte do Conselho Diretor e dos membros da SBM.

I. Substituir o logo pela espiral tangente no retângulo $\ell_{0} \times 1$.

II. Substituir o logo pela espiral tangente na página e nas publicações digitais da SBM como uma animação explorando as propriedades da espiral logarítmica ${ }^{9}$.

${ }^{8}$ Observação devida a Humberto Bortolossi.

${ }^{9}$ Ideia sugerida por Paulo Cézar Carvalho. 

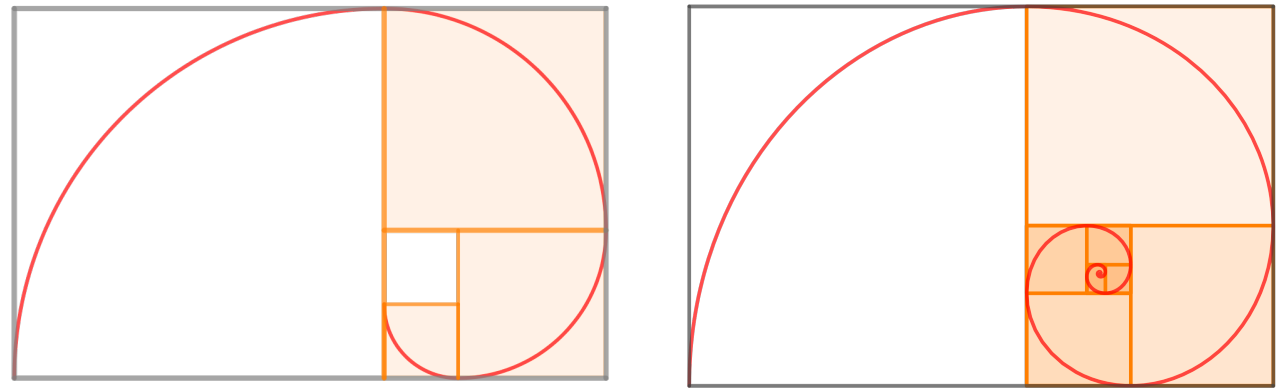

\section{AgradeCIMENTos}

O autor agradece a generosa colaboração de César Camacho, Humberto Bortolossi, Marco Moriconi e Paulo Cézar Carvalho, bem como a dos dois relatores.

\section{REFERÊNCIAS}

[1] Elon Lages Lima, Paulo Cézar Pinto Carvalho, Eduardo Wagner e Augusto César Morgado: A Matemática do Ensino Médio. Coleção do Professor de Matemática, SBM, 1996

[2] Raymond Claire Archibald: Notes on the logarithmic spiral, golden section and the Fibonacci series. Note V in Jay Hambidge: Dynamic symmetry. Yale University Press, 1920.

[3] Øyvind Hammer: The Perfect Shape: Spiral Stories. Copernicus, 2016.

[4] Herman Erlichson: Newton's Solution to the Equiangular Spiral Problem and a New Solution Using Only the Equiangular Property. Historia Mathematica 19 (1992), pp.420-413.

[5] Curtis Wilson: Newton on the Equiangular Spiral. An Addendum to Erlichson's Account. Historia Mathematica 21 (1994), pp.196-203.

[6] Sérgio Alves: A espiral equiangular. Notas de minicurso, I Bienal da SBM, 2002.

[7] Eli Maor: The logarithmic spiral. The Mathematics Teacher, vol. 67(4), 1974, pp. 321-327.

[8] Kinko Tsuji, Stefan Müller (eds): Spirals and vortices in Culture, Nature and Science. Springer Verlag, 2019.

[9] Jay Kappraff: The Logarithmic Spiral in Geometry, Nature, Architecture, Design and Music. https://directorymathsed.net/public/CataniaConferenceDocuments\& Papers/Proceedings/Al1\%20PapersInPDF/KAPPRAFF.pdf

[10] Khristo Boyadzhiev: Spirals and Conchospirals in the Flight of Insects. The College Mathematics Journal 30(1), 1999, pp. 23-31.

[11] Jim Wilson: Equiangular Spiral (or Logarithmic Spiral) and Its Related Curves. http://jwilson.coe.uga.edu/EMT668/EMAT6680.F99/Erbas/KURSATgeometrypro/ related\%20curves/related\%20curves.html

[12] https://mathcurve.com/courbes2d.gb//logarithmic/logarithmic.shtml

[13] http://xahlee.info/SpecialPlaneCurves_dir/EquiangularSpiral_dir/ equiangularSpiral.html

[14] http://www.spirasolaris.ca

[15] Arthur Lee Loeb \& William Varney: Does the golden spiral exist, and if not, where is its center? In István Hargittai \& Clifford Alan Pickover (eds.): Spiral Symmetry, World Scientific, 1992.

[16] John Sharp: Spirals and the golden section. Nexus Network Journal, vol. 4(1), 2002, pp. 59-82.

[17] Viviane de Oliveira Santos: Uma história da Sociedade Brasileira de Matemática durante o período de 1969 a 1989: criação e desenvolvimento. Tese de doutorado, Unesp - Rio Claro, 2016.

[18] Manual da marca e identidade visual. SBM, 2017. https://www.sbm.org.br/wp-content/ uploads/2017/03/SBM_Manual_Marca_ver20170223.pdf 
[19] https://en.wikipedia.org/wiki/Fibonacci_number

[20] Andrey Polezhaev: Spirals, Their Types and Peculiarities. In Kinko Tsuji, Stefan Müller (eds): Spirals and vortices in Culture, Nature and Science.

[21] The Fibonacci Sequence in Nature. https://insteading.com/blog/ fibonacci-sequence-in-nature/

[22] The Fibonacci Sequence Is Everywhere - Even the Troubled Stock Market. https://www. smithsonianmag.com/science-nature/fibonacci-sequence-stock-market-180974487/

[23] The Fibonacci Sequence In Artistic Composition. https://www.markmitchellpaintings. $\mathrm{com} / \mathrm{blog} /$ the-fibonacci-sequence-in-artistic-composition/

[24] Nature's proof of inteligent design: sacred geometry, phi, the Fibonacci spiral, 83 self-reflective designs. https://newearthknowledge.com/2019/11/22/proof-intelligent-design/

[25] https://en.wikipedia.org/wiki/Golden_spiral

[26] Branko Grunbaum: Geometry strikes again. Mathematics Magazine 58(1) (Jan.1985), pp. 12-17.

[27] Doris Schattschneider: The mistery of the MAA logo. Mathematics Magazine 58(1) (Jan.1985), p. 18

Rua da Nascente 136, Distrito de São Gonçalo do Rio das Pedras, Serro, MG Email address: michelspira@gmail.com 